of $a C_{i}$ and $R^{3}$ determine thus $3 i(3 m-1)$ such points which can be arranged in primary sets $S^{\prime}{ }_{3 i}$ of the $3 i$ points in $(3 m-1)^{3 i-1}$ ways.

Passing now to the general cubic, if a $C_{m}$ have $(3 m-1)$ point contact at $u^{\prime}$ and cuts again at $u$ the relation connecting the elliptic arguments is

$$
u^{\prime}=\frac{\mu \omega+\mu^{\prime} \omega^{\prime}-u}{3 m-1} .
$$

For given $u$ there are $(3 m-1)^{2}$ values of $u^{\prime}$ since $\mu, \mu^{\prime}=1$, $2, \cdots, 3 m-1$. Thus from the common points of a conic and a $C_{3}$ can be drawn $6 \cdot 25$ quintactic conics whose 150 quintactic points lie by sixes on $5^{10}$ primary conics.

And generally from the $3 i$ common points of $a C_{i}$ and $C_{3}$ can be drawn $3 i(3 m-1)^{2} C_{m}$ 's each having a $(3 m-1)$-point contact. These $3 i(3 m-1)^{2} P_{3 m-1}$ 's can be grouped in primary sets $S_{3 i}{ }^{\prime}$ of the $3 i$ points in $3(3 m-1)^{6 i-2}$ ways.

UNIVERSITY OF WASHINGTON.

\title{
CAJORI'S HISTORY OF MATHEMATICS
}

A History of Mathematics. By Florian Cajori, Ph.D., Professor of the History of Mathematics in the University of California. New York, The Macmillan Company, 1919. vii +514 pp. Price, $\$ 4.00$.

THE present edition of Cajori's well known History of Mathematics is so completely revised and so considerably enlarged that it might almost be regarded as a new book. While it contains only about 100 more pages than the earlier edition,* it has about twice as much reading matter, the pages being larger and more closely printed than those of the first edition. The general arrangement of the subjects treated remains unchanged, but three brief new sections relating largely to ancient mathematics have been added. These are headed; "The Maya," "The Chinese" and "The Japanese" respectively, and are based on special histories relating to these peoples, which have appeared since the publication in 1894 of the first edition of the present work.

* Reviewed in this Bulletin, vol. 3 (1894), pp. 190 and 248, by D. E. Smith and G. B. Halsted. 
Naturally the most important and extensive changes appear in the sections devoted to developments made during the nineteenth century. Somewhat less than one half of the space is devoted to advances made during the nineteenth and the early part of the twentieth century. To the younger mathematical students it is likely to be very interesting and useful to find here references to the work of so many contemporary mathematicians, and to find also brief statements of the relations between contemporary developments and those due to earlier investigators.

In view of the great variety of modern mathematical advances it cannot be expected that all of them could be treated in a one-volume history. The selection of the material to be included in such a volume was a very difficult undertaking and few would be likely to agree as regards details in making such a selection. In a broad way, however, probably most mathematicians will agree that Cajori succeeded very well in selecting his material and that he treated this material with an evident effort to exhibit relative values in a true light.

To secure reliable judgments as regards the bearing and value of much of the modern work, Cajori had to depend largely on the views of specialists. He quotes these views freely and sometimes extensively. The lack of uniformity in style and conservatism thus introduced is more than compensated by the expert knowledge relating to the wide range of fields of investigation which is thus made available. As a rule this expert knowledge has been wisely utilized and references are furnished. In some cases the latter are lacking but the total number of references is large and in this respect the present edition exhibits also a great improvement on the former one.

Two important questions about a history of mathematics are: Is the work written in an attractive style? and Is it confined to established facts? In the present case our answer to the former question is yes, and to the latter, no. Our negative answer to the second of these two questions does not imply a criticism of the book, since many readers will doubtless enjoy such speculations as those in regard to the reason why the ancient Babylonians divided the circle into 360 equal parts, page 6 , as well as the references to reports which are as unlikely to be true as the one relating to the sacrifice of one hundred oxen by Pythagoras, page 18. 
The value of a comparatively brief general history of mathematics cannot be measured entirely by its accuracy as regards details. The field to be covered is so extensive and the number of data is so large that it is almost impossible to avoid entirely the appearance of some misleading statements. What is most important is that the main points of view are sound and that the most fundamental factors in the development of our subject are properly emphasized.

The present work appears to be satisfactory along these important lines, as might have been expected from the fact that its author has had such a broad training in writing on the history of mathematics. Many historical questions present great difficulties and call for a wide knowledge of facts as well as a deep insight into the bearing of one result on another and the tendencies, local as well as national, to claim undue credit for the work of particular investigators.

It is interesting to note that in the present edition the word khet is used in place of ruth to denote an ancient Egyptian unit of unknown length employed in the work of Ahmes. The introduction of the term ruth for this unit seems to be due to a very crude error committed by J. Gow in his History of Greek Mathematics, 1884, page 127. In making use of A. Eisenlohr's translation of the work by Ahmes, Gow apparently failed to notice that the German word Ruthen means rods, and hence he translated this word by the word ruths instead of by the word rods.

The error thus committed found its way into other histories as well as into textbooks. It appears, for instance, on page 44 of the earlier edition of the present work as well as in the revised edition of Cajori's History of Elementary Mathematics, published in 1917. Fortunately, it is corrected in the present work and it is hoped that this correction may help to banish this error from the future textbooks on elementary geometry. In making the correction Cajori wisely transliterated the ancient Egyptian word instead of attempting to give an English equivalent.

Those who are especially interested in American mathematical work will be pleased to find so many references in the volume under review to the work done by Americans. By glancing over the Index it becomes at once apparent that America is on the mathematical map,-a fact which is not always revealed by the Index of a mathematical history. On 
the other hand, one fails to find in this Index as large a number of references after the name of an American as appears after the names of various foreign mathematicians. This difference does not apply only to the deceased mathematicians, although it is more pronounced in these cases. In special instances such differences may be more or less due to accident, but when wide differences of this kind present themselves as regard large collections they should not be attributed to accident and their significance becomes a matter for serious consideration.

The semi-biographical feature of the earlier edition has been retained in the present one, as well as the use of heavy type in printing the names of some of the most eminent mathematicians. The following eight names of Americans appear in such type: M. Bôcher, J. W. Gibbs, G. M. Green, G. W. Hill, E. McClintock, A. Macfarlane, S. Newcomb, and B. Peirce. While no names of living American mathematicians are printed in this way, such a restriction has not been observed in regard to European mathematicians, as the following names appear in heavy type: P. Appell, P. Bachmann, E. I. Fredholm, G. Frege, F. Klein, H. Lebesgue, K. Pearson, E. Picard, H. A. Schwarz. Several of these names could have been replaced by those of more eminent living investigators.

One of the most useful things that the reviewer of a new edition of a popular textbook can do is to direct attention to possible improvements in such a way as to be helpful to those who will use the book in the class-room. It is with this object in view that we make the following suggestions: The note on page 98 relating to our lack of knowledge as regards the origin of our common numerals appears to the reviewer to be correct, but it does not seem to be in accord with a number of statements appearing in other parts of the book, e.g., pages 55, 88, 97, and 100 . It seems as if this note had been added after it was too late to revise the other statements relating to this subject. If this is the case the note could have been made much more effective by stating this fact therein even if it might appear as acknowledging that the book was in need of a revision before it was published.

On page 27 it is stated that "Menaechmus invented the conic sections," and on page 39 we read that "Menaechmus, and all his successors down to Apollonius, considered only sections of right cones by a plane perpendicular to their sides." The latter of these two statements seems to be especially 
misleading, since both Euclid and Archimedes knew how to generate an ellipse by cutting the cone by a plane not perpendicular to a generator. As regards the former statement, F. Dingeldey remarked that it is probably impossible to establish the correctness of the view that Menaechmus was the discoverer of conic sections and that it is very questionable whether he knew that the curves in question can be obtained by cutting a cone by means of a plane (Repertorium der höheren Mathematik, volume 2 (1910), page 197; Encyclopédie des Sciences Mathématiques, tome 3, volume 3, page 41).

That Cajori's language is not always clear and direct may be illustrated by the following quotation from page 47: "The fundamental theorem of plane trigonometry, that two sides of a triangle are to each other as the chords of double the arcs measuring the angles opposite the two sides, was not stated explicitly by Ptolemy, but was contained implicitly in other theorems." It is difficult to see why such cumbersome language is employed here to express the well-known law of sines in regard to the plane triangle. If the object was to illustrate the language which Ptolemy might have employed to express this law, reference should have been made to this fact.

Other instances of a lack of clarity or of obvious misprints may be found as follows:

$\begin{array}{rrrrrc}\text { Page. } & \text { Line. } & \text { Page. } & \text { Line. } & \text { Page. } & \text { Line. } \\ 49 & 31 & 299 & 18 & 372 & 39 \\ 239 & 27 & 302 & 16 & 373 & 34 \\ 250 & 21 & 327 & 1 & 377 & 20 \\ " & 22 & 330 & 10 & 401 & 32 \\ 265 & 31 & 331 & 8 & 418 & 22 \\ 267 & 33 & 351 & 35 & 438 & 15 \\ 287 & 3 & 360 & 33 & 494 & 42 \\ 296 & 6 & 361 & 26 & 497 & 37\end{array}$

To test the Index as regards completeness, the places where the term "group" would be likely to appear were looked up by the reviewer and it was found that the following page numbers should be added to those following this term in the Index: $318,323,390,432$. On the other hand, the term group does not appear on any of the pages 362-366 and hence the series of numbers $349-366$ following this term in the Index should be replaced by 349-361. The name of $\mathrm{H}$. Lebesgue does not appear in the Index, although it is printed in heavy type in the text, as was noted above. 
It is singular that the Index does not contain the term "fundamental theorem of algebra." This term appears on page 363 of the text and reference is made in the Index to the substance of the theorem under the term "roots." To this reference there should be added the number 253 where the following sentence appears: "In the Résolution des équations numériques (1798) he (Lagrange) gave, among other things, a proof that every equation must have a root, - a theorem which before this usually had been considered self-evident."

This statement is clearly misleading, since various demonstrations of the theorem had been attempted long before 1798. In particular d'Alembert published such a supposed demonstration in 1746 and the theorem is still frequently called D'Alembert's theorem, especially in France. Among others who had attempted to give demonstrations before 1798 is L. Euler, who furnished two such demonstrations in 1749. In fact, Lagrange had also attempted to give such demonstrations more than twenty years before 1798 . The great historical importance of the fundamental theorem of algebra makes it especially desirable that the references relating thereto should be explicit and accurate.

On page 234 we read "the calculus of variations to the invention of which Euler was led by the study of the researches of Johann and Jakob Bernoulli." On the other hand, the following sentence appears on page 251. "Lagrange did quite as much as Euler towards the creation of the Calculus of Variations." If Lagrange did quite as much as Euler towards the creation of this subject it is difficult to see why Euler should be regarded as its inventor.

The lack of uniformity in the two statements noted in the preceding paragraph would be less objectionable if Euler were commonly regarded by the best modern writers as the founder of the calculus of variations. On the other hand, the vagueness involved in the term inventor of a subject and the disagreement among historical writers as to who should be regarded as the founders of various subjects tend to mitigate somewhat such apparently contradictory statements as those noted above.

There is a very noticeable lack of uniformity as regards names. That both of the names Petrograd and St. Petersburg are used for the same city is perhaps due to the somewhat recent change of its name. This is less confusing 
than the adoption of spellings according to different languages of the name of the same city and the use of different initials in referring to the same person at different places. For instance, those acquainted with the works of E. Lucas will be apt to be somewhat annoyed to find his name also in the form François Edouard Anatole Lucas. It is questionable whether anything is gained by giving G. Frege's name in the form Friedrich Ludwig Gottlob Frege.

The question of form as regards names in references and in historical works presents serious difficulties in view of the lack of uniformity along this line on the part of good writers. It is frequently very undesirable to give only the family name since the number of family names represented by different mathematicians is already large and is constantly growing larger. On the other hand the names in full are often so lengthy as to become burdensome. Perhaps the most satisfactory solution is to follow the example of the large mathematical encyclopedias. At any rate, it would be desirable to have uniformity in the same work unless an explanation is made as regards the equivalent different forms used in such a work.

Since B. Peirce occupies such a prominent place in the history of American mathematics it may be of interest to quote the following from page 338: "Profound are his researches on Linear Associative Algebra. The first of several papers thereon was read at the first meeting of the American Association for the Advancement of Science in 1864." The American Association for the Advancement of Science did not hold any meeting in 1864, its first meeting was held in 1848, and its Proccedings for this meeting do not mention any paper on linear associative algebra.

These suggested improvements may serve to direct attention to the fact that the careful student of the history of mathematics will be inclined to make a large number of marginal notes in Cajori's book and should be encouraged to do so. On the other hand, he will find here a very useful and readable history of our subject. The numerous quotations from reliable sources, the large number of references to the work of contemporary mathematicians and the fine spirit which pervades the whole work tend to increase the value of the book. It is the largest and most modern general history of mathematics in our language and we wish for it a success commensurate with the large amount of labor involved in its preparation. 